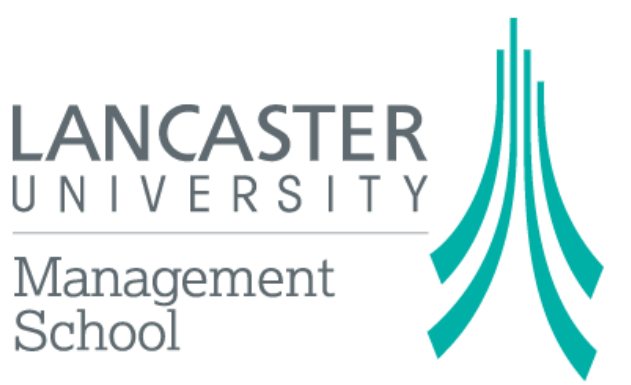

Economics Working Paper Series

2014/015

\title{
Traffic Accidents and the London Congestion Charge
}

Colin P. Green, John. S. Heywood and Maria Navarro

The Department of Economics Lancaster University Management School

Lancaster LA1 4YX

UK

All rights reserved. Short sections of text, not to exceed two paragraphs, may be quoted without explicit permission, provided that full acknowledgement is given. 


\title{
Traffic Accidents and the London Congestion Charge
}

\author{
Colin P. Green ${ }^{1}$, John. S. Heywood ${ }^{2,1}$ and Maria Navarro ${ }^{1}$ \\ ${ }^{1}$ Lancaster University \\ ${ }^{2}$ University of Wisconsin - Milwaukee
}

\begin{abstract}
In a rare effort to internalize congestion costs, London recently instituted charges for traveling by car to the central city during peak hours. Although the theoretical influence on the number and severity of traffic accidents is ambiguous, we show that the policy generated a substantial reduction in both accidents and fatalities in the charged area and hours. At the same time, the spatial, temporal and vehicle specific nature of the charge may cause unintended substitutions as traffic and accidents shift to other proximate areas, times and to uncharged vehicles. We demonstrate that, to the contrary, the congestion charge reduced accidents and fatalities in adjacent areas, times and for uncharged vehicles. These results are consistent with the government's objective to use the congestion charge to more broadly promote public transport and change driving habits.
\end{abstract}

JEL Codes: I18, R48, H27

Keywords: Traffic Congestion; Pricing; Vehicle Accidents

The authors thank the Melvin Lurie Memorial Fund at UWM which allowed Heywood and Navarro to work together in the fall of 2013. The authors thank Domingo Perez-Ximenez de Embun for helpful comments. 


\section{Introduction}

Early in 2003 London imposed a daily charge for driving on public roads within its central district. Economists hailed the charge as "a triumph of economics," a recognition by policy makers that congestion is a costly externality and that road pricing is an appropriate response (Leape 2006). While the charge remains flat and so does not vary with distance or time of day, it has been credited with substantial reductions in congestion and increases in travel speed. Less examined is the influence on traffic accidents. While reduced traffic accidents were touted as an additional social benefit, the policy created a series of offsetting behavioral incentives that leave the overall influence on traffic accidents in doubt. Examining this influence requires suitable counterfactuals as the number of London traffic accidents had been trending down prior to the congestion charge.

This paper examines monthly traffic accident counts in central London before and after the congestion charge compared to several suitable controls. We confirm a substantial and robust decline in accidents associated with the advent of the congestion charge. As the charge is limited to a specific zone, for specific vehicles and for specific hours of the week, we test for substitution effects. These measure the extent to which the charge may increase accidents in areas outside the zone, the vehicle type or the hours to which it applies. Such increases might be anticipated if travelers continue to come to Central London but substitute uncharged trips for charged trips. Thus, we examine whether or not traffic accidents increase on weekends and evenings (times not subject to the charge). We examine whether or not accidents increase for motorbikes, bicycles or taxis which are all exempt. Finally, we investigate whether accidents increase in areas immediately adjacent to the charge zone as previous through drivers skirt the charge zone or as drivers travel up to the zone and then cross onto public transport. We find no 
evidence of long-term accident increases in any of these three dimensions. Indeed, traffic accidents decline in adjacent areas, out of charged times and for uncharged vehicles relative to controls. This contradicts earlier evaluations that fail to use suitable controls and examine only a shorter window for policy influences. We also confirm that the decline in total accidents in the charged zone and time is matched by a decline in serious accidents and in fatalities. These declines also persist in proximate regions and uncharged times. In sum, the evidence suggests that the congestion charge helps in accomplishing the government objective of fundamentally changing behavior regarding the frequency and mode of transit into Central London with beneficial and general reductions in the number of traffic accidents, a point not previously made.

\section{Background}

Central London has long held a reputation as among the most congested of major Western cities. Over the second half of the twentieth century, traffic speeds decreased and vehicle counts increased. Just prior to imposing the charge, all-day average network travel speeds averaged a sluggish $8.6 \mathrm{mph}$ and more than 1/3 of all travel time was spent simply not moving (Transport for London 2003). When compared to an uncongested speed of around 20 miles per hour, this represented 3.7 minutes per mile of lost time. Multiplied by the huge number of trips and the value of time, the waste was obviously enormous. Fully ninety percent of all London residents (not just those of Central London) agreed in polls that "there is too much traffic in London" and identified congestion as the "most important problem requiring action" (see survey description and references in Leape 2006, p. 157).

At least since Pigou (1924), economists have advocated governmental taxes and charges to bring the actual prices that consumers face into alignment with full social costs. The 
application of this notion to congested roads dates back to at least Walters (1961) and Vickrey (1963) who emphasize that consumers should pay directly for the costs they impose on other travelers as an incentive to use road resources efficiently. If road space is unpriced, traffic volumes will increase until congestion limits further growth with a resulting waste in travel time and reduction in travel reliability. Additional costs associated with congestion include increased air pollution and increased energy dependence (see Parry et al. 2007). Despite the advantages of taxing congestion, there exists a long history of public and political opposition that has meant there have been relatively few examples (Harsman and Quigley 2010).

In addition to political resistance, network issues make proper pricing inherently difficult. While pricing a single road between two destinations may be easy, properly pricing for a complicated road network like Central London was thought unworkable. Each intersection, road and specific set of combinations contributes to congestion. Moreover, each of these contributes in differing degrees at different times of the day, week or year. Thus, while optimal charges vary by road, intersection and time of day, the creation and enforcement of such charges is likely intractable or infeasible (Newberry 1990; Shepherd and Sumalee 2004). Moreover, the proper pricing may interact in complicated ways with the extent and pricing of parking (Fosgerau and de Palma 2013) and the endogenous choice of speed by drivers (Verhoef and Rouwendal 2004). Thus, the London congestion charge emerged as a rather blunt instrument. It followed the basic approach "to make private transport relatively less attractive and public transport more attractive.” (Newberry 1990 p. 35) It combined a flat charge for private and commercial vehicles entering the congestion zone, with the revenues from the charge earmarked for reinvestment in London's public transport. 
London imposed an initial daily charge in February 2003 of $£ 5$ for driving on roads within the congestion zone between 7:00 am and 6:30 pm on weekdays. ${ }^{1}$ The congestion zone is pictured in Figure 1. The original fee has since been increased twice to £8 in July of 2005 and to $£ 10$ in 2011. Passes are typically purchased on-line and enforcement relies on a series of video cameras at every entry point to the zone and on mobile units within the zone. A license plate recognition system matches against daily purchases and violators are sent penalty notices for escalating fines that average 20 to 30 times the daily charge. The day pass allows travel in and around the congestion zone of Central London. This eight square mile zone includes tourist sites, the City (London's financial district), Parliament, major government offices and prime business locations.

\section{Insert Figure 1}

The charge applies to private and commercial vehicles entering the congestion zone during the charging hours. Importantly, motorcycles, bicycles, buses and taxis are exempt. Also exempt, are vehicles belonging to those who live within the zone but keep their vehicles off the street during the charging hours. When these residents do travel during the charging hours, they pay a highly discounted charge of only 10 percent of the full charge.

The revenue raised from the charging program has been substantial but so have the administrative costs (Leape 2006). The net revenue from charges was $£ 97$ million in 2004-5 and was supplemented by £70 million in penalties that same year. Such revenues have been largely spent on mass transit improvements with smaller expenditures on road safety and biking/walking

\footnotetext{
${ }^{1}$ Beginning in February 2007, the end of the charge time was moved from 6:30 pm to 6:00 pm, a move we account for explicitly in identifying accidents in the treatment.
} 
initiatives. The earmarking of revenues for such alternative transport is anticipated to continue until at least 2023.

Early indications showed meaningful reductions in distances traveled within the zone. These comparisons of the year immediately before and after the charge showed, for example, that the total distance driven by cars was reduced by an enormous 34 percent (Leape 2006). At the same time, the distances driven by bikes, motorcycles, taxis and buses all increased resulting in a more modest overall decline in vehicle distances of 12 percent. Nonetheless, this was sufficient to reduce the time lost to congestion by nearly 30 percent (Transport for London 2005). Thus, the early indication was clear that the charge reduced congestion during the times it was applied, in the zone to which it applied, and for the vehicles to which it applied. This generates substantial social benefits as the values placed by individuals on reduced travel time and improved reliability are typically large (Small, Winston, and Yan, 2005).

In addition to reducing congestion and so saving time, a critical by-product of the charge was thought to be reduced traffic accidents. While clearly identified as "an additional social benefit" by Transport for London (2005), the logic implying an overall reduction in accidents seems in doubt. First, Shefer and Rietveld (1987) argue that there should be an inverse relationship between traffic congestion and accidents. The increase in speeds allowed by reduced congestion may increase the number and severity of accidents. Certainly, this balancing of time savings and the increased chance of traffic fatalities is at the heart of setting speed limits (Ashenfelter and Greenstone 2004). While the evidence seems to depend on the exact circumstances and perhaps even the type of roads being examined (Wang et al. 2009), the possibility exists that the congestion charge increased vehicle speed and at the same time 
increased the number of bikes and pedestrians with an uncertain net influence on the number and severity of accidents.

Second, even if the congestion charge reduced the number of accidents by reducing the trips by those charged, there are important avenues of substitution. In the empirical estimation we focus on three forms of substitution. Most fundamentally, those who would otherwise be charged may substitute the nearest uncharged route. As Parry and Benito (2002) emphasize, charging on one route or in one area may simply add to congestion elsewhere and in a complex network it may not be possible to monitor and charge all of these spillovers. Thus, cross traffic that might have gone directly through Central London can be expected to avoid the charge zone but increase congestion in adjacent areas. Commuters might be anticipated to drive up to the charge zone and search for parking before crossing into the zone without their vehicle. Parry and Benito (2002) argue that the increased congestion in alternative areas will increase traffic accidents in these uncharged adjacent areas. Second, those who would otherwise be charged may substitute to uncharged vehicles. As mentioned, bikes, motorbikes and taxis are exempt. ${ }^{2}$ Third, those who would otherwise be charged may substitute out of the weekday charge time by rearranging trips to the evenings or weekends. While not every trip might be easily shifted, it seems sensible for a variety of shopping, entertainment and social trips. Thus, in addition to examining the pattern of accidents in the charge zone during the charge time and for the charged vehicles, we will test for the extent of substitution on these three important margins.

Others have been concerned with the influence of the congestion charge on traffic accidents. Early comparisons simply examined numbers of accidents in the charging zone before and after the charge (Leape 2006; Quddus 2008). As suggested, this may be problematic both

\footnotetext{
${ }^{2}$ Indeed, motorbike dealers ran advertising campaigns encouraging commuters to purchase their product with the slogan "make Mayor Livingston see red," as motorbikes would not be charged and so not contribute to the profitability of the congestion charge.
} 
because of substitution out of the zone and also because the trend was of decreasing accidents within the zone prior to introducing the charge. Li et al. (2012) examine a particularly short time frame and show a decrease in car casualties within the zone relative to those happening in the English city of Leeds. For motorcycle casualties they find an increase in London compared to Birmingham and, similarly, for bicycle casualties they find an increase in London compared to Manchester.

We provide a comprehensive examination of the influence of the congestion charge that examines all accidents as well as serious and fatal accidents. We explore how robust the results are to choice of the control, empirical specification and to lengthening the time frame. We examine the influence of the congestion charge on charged vehicle accidents within the charge zone and hours. We then investigate the influence of the congestion charge on adjacent regions, times and on uncharged vehicles.

\section{Data and Methodology}

We use road accident data from the Department of the Environment, Transport and the Regions (DETR) that contain all motor vehicle accidents reported to the police from 2000 to 2010 for all 416 local jurisdictions in Britain. ${ }^{3}$ We know the type of accident (whether it caused either serious injury or death), the date and time of the accident, location of the accident and the age of the driver of any vehicle involved in the accident. This, when combined with GIS mapping of the congestion zone, allows us to accurately assign accidents to the congestion charge zone in the pre and post policy periods. In addition this allows us to assign accidents to areas that are adjacent to but outside the congestion charge zone (CCZ).

\footnotetext{
${ }^{3}$ Available from the UK data archive.
} 


\section{INSERT FIGURE 2}

Figure 2 provides initial evidence of the congestion charge effect on traffic accidents in the CCZ. We use as an initial comparison the average monthly accidents per city for the 20 most populous cities in Great Britain (excluding London). The figure shows the accidents in charged times for charged vehicles for both the CCZ and the control group. It demonstrates a declining trend in accidents over the period and shows evidence of seasonality, both of which are well known features of traffic accidents in Great Britain and will be controlled for in our estimations. Otherwise the comparison series appears reasonably stable before and after the congestion charge. In contrast, the monthly accidents in the congestion charge zone drop markedly after the congestion charge. Initially accidents in the CCZ are approximately 40 higher per month than the comparison group. This difference essentially disappears after the introduction of the charge.

\section{INSERT FIGURE 3}

Figure 3 brings these points into sharper relief. It provides linear spline estimates of traffic accidents before and after the introduction of the congestion charge for the CCZ and the comparison group. There is a large reduction in accidents in the CCZ that is coincident with the introduction of charging with reasonably similar trends either side of the change. For the control group there is no evidence of a level change at the discontinuity nor is there a clear change in trends.

The initial specification estimates the number of accidents per jurisdiction and month in a difference in difference formulation:

$$
A c c_{i t}=\phi+\delta C C Z_{i}+\alpha \text { Policy }_{t}+\beta\left(C C Z_{i} * \text { Policy }_{t}\right)+\gamma X_{i t}+\tau T_{t}++\varepsilon_{i t}
$$


In this specification Acc is the number of accidents in the month and area (there are 21 areas, the twenty largest cities and the CCZ), CCZ indicates that the accident was within the congestion charge zone, Policy indicates that the accident happens after the date of the congestion charge policy, $T$ is a linear time trend, $X$ a vector of controls. The key parameter of interest is $\beta$ which provides the difference in difference estimate of the effect of the congestion charge on accidents.

Several empirical challenges exist when identifying the effect of the congestion charge on accidents. It is well known that during our period of analysis, traffic accidents and fatalities have generally been declining in England and in central London (Department of Transport, 2012). We will explore the underlying parallel trends assumption in a flexible version of (1) where time trends are allowed to vary between the treatment and control. We begin by contrasting the congestion charge area, time and vehicles to the controls of the 20 largest cities in Britain for the charge time and vehicles. We cluster standard errors at the jurisdiction level but ultimately experiment with this as well suggesting that the pattern we identify is robust.

We then move beyond this to allow the data to determine a synthetic control that optimally weights the various 20 cities to match the underlying characteristics of the treated CCZ (Abadie and Gardeazabal 2003 and Abadie et al. 2010). The matching process minimises the mean squared prediction error (the average number of accidents per jurisdiction in the CCZ minus that in the synthetic control) for the pre-policy periods. The resulting control exhibits the most similar traffic accident pattern to that observed in the CCZ before the passage of the congestion charge and is then compared to the CCZ in a straightforward difference in difference.

The likelihood that the congestion charge will have influenced traffic patterns in neighboring areas leads us to remove all other areas of London from our control group from the start. In subsequent analysis we explicitly seek to examine these geographic spillovers among 
other types of spillover. We will also separately focus on serious and fatal accidents and explicitly consider other related policy changes during our data window.

\section{Empirical Results}

The first column of Table 1 provides a difference in difference estimate of the policy effect in an initial estimate of (1). This includes a single trend variable revealing the downward trend in accidents and adds quarterly dummies to capture the evident cyclical pattern seen in the raw data. In a pattern, often noted in the British data, the fourth quarter is found to have the highest number of accidents (the last three months of each calendar year). The estimate reveals that the congestion charge is associated with 30 fewer accidents per month in the CCZ when compared to the other 20 cities. Figure 2 provided a modest suggestion that there could be different pre-trends in the control jurisdictions and the CCZ. The second column allows for differential trends between treatment and control. The interaction term between CCZ and the monthly trend is not statistically significant and the underlying trend estimate remains largely unchanged. Most critically, the estimate of the congestion charge effect also remains essentially unchanged. In Appendix Table 1 we add controls for area and population of the underlying jurisdictions which suggest that while these are associated with increased accidents, their inclusion does not eliminate the evident policy influence.

\section{INSERT TABLE 1}

The underlying accidents within a given area can be viewed as generating a count variable. This may have implications for both our point estimates and their precision. The next two columns of Table 1 examine this by estimating both Poisson and negative binomial models 
of accidents. We note that there is no concern with zero inflation as none of the jurisdictions have a single period with zero accidents. The results mirror those already presented as they show statistically significant and large reductions in the number of accidents associated with the introduction of charging. These reductions of $38-40 \%$ correspond broadly with the magnitude from the OLS estimates. The null of no overdispersion of the dependent variable is rejected at the 1 per cent level. Thus, the model is more correctly estimated via negative binomial than Poisson. In an effort to determine whether we should continue to use the negative binomial, we calculated the mean squared residuals for both the negative binomial and the original linear specification in column (2). They were very similar but that for the linear specification did slightly better (1154.4 vs. 1155.9). Critically, we found no specification in which the linear estimate returned a significant policy reduction but the negative binomial did not. Thus, in subsequent estimates we focus on OLS estimation but will sometimes provide the percentage measure from the negative binomial for ease of comparison. ${ }^{4}$

An additional concern may be that identification of the key parameter comes from a change in policy by a small number of groups (one single local authority) in a relatively small number of overall groups. Clustering at the local authority level in this case can cause the reported standard errors to be misleadingly small. In response we return to the estimate in column 2 and implement the Wild bootstrap procedure from Cameron et al. (2008). This dramatically reduces the high type I error rates common in the presence of clustering on a small number of groups. The procedure replicates the within group correlation in errors when generating new estimates. Under the null hypothesis of no difference in difference effect, the Wild bootstrap p-value clustered at a local authority level with 1000 replications is 0.032

\footnotetext{
${ }^{4}$ We also estimated both a simple logistic estimate and a linear estimate that controlled for jurisdiction fixed effects with no meaningful change in either significance or magnitude.
} 
rejecting the null. As a second approach, we follow Conley and Taber (2011) who suggest an alternative inference procedure based on the distribution of residuals across all jurisdictions that performs better than clustered standard errors when there are very few treated groups. The $90 \%$ confidence interval formed by using information from the larger sample continues to exclude a treatment effect of zero. The sum of these two tests suggests that the observed difference-indifference represents a genuine influence.

One feature of the data illustrated in Figures 2 and 3 is the large differences in average accident levels between the CCZ and other cities in the Great Britain. This reflects the unique position of central London in terms of activity and traffic density. This might cause concern regarding the suitability of our control group. To address this we adopt the synthetic panel approach as set out by Abadie and Gardeazabal (2001) and Abadie et al (2010). This involves optimally weighting the comparison group to match the pre-treatment accident data for the CCZ. As a result of this weighting, the mean squared prediction error between the CCZ and the control was reduced from over 1000 using the 20 largest cities to only 20.4 with the optimal weighting of those cities. All cities took a positive weight in the optimal match although many received only a couple of percentage points. The largest weighting was given to Birmingham (the second largest British city) with .259. Next in order were Leeds, Manchester and Liverpool, each receiving between .05 and .10 .

\section{INSERT FIGURE 4}

The result of the matching is demonstrated in Figure 4. This shows a very close match between the pre-accident levels and trends for the CCZ and synthetic control group, followed by a marked reduction in accidents post charge introduction. The corresponding point estimates 
from the difference-in-difference are reported in the final column of Table 1. These suggest an effect of congestion charging that is again in the order of a 34 accident reduction per month. Thus, the differences between a not weighted and optimally weighted control appear modest. Critically, the fact that the optimal weighting scheme includes all cities indicates that it is superior to simply using a single alternative jurisdiction as the control (as done in Li et al. 2012).

\section{The Spillover Effects of Congestion Charging}

The prior analysis clearly indicates that the congestion charge reduced accidents involving treated vehicles within the congestion zone and time. Yet, these estimates may dramatically differ from the full influence of the charge. The estimates presented may overstate the full influence if traffic moves into uncharged times, regions or vehicles. In the extreme, one might fear that accidents are simply displaced and not truly reduced rendering the previous estimates largely meaningless. The alternative is that the policy influence identified earlier spillovers over actually reducing accidents in adjacent regions and times. This seems at least plausible as the charged zone is at the center of a wheel and spokes. It thus eliminates vehicle trips that would have come into the central district only after crossing many of the adjacent areas. Moreover, an explicit objective of the congestion charge zone policy was to encourage broader use of mass transit and this increased use could carry over to times outside the charged hours and areas. ${ }^{5}$ Thus, we test for the full influence of the congestion charge by measuring the substitution effects, the extent to which the charge influences accidents in areas outside the zone, the vehicle type or the hours to which it applies.

\section{INSERT TABLE 2}

\footnotetext{
${ }^{5}$ Recall that the net revenue from congestion charge is earmarked to improve mass transit.
} 
Table 2 estimates variants of model (2) from Table 1 (i.e. difference in difference allowing for differential trends) for potential margins of substitution. First, we use GIS to identify all accidents outside the CCZ but within 2 kilometers of the CCZ boundary. We identify this as spillover region 1 . We then identify all accidents outside the CCZ and outside spillover region 1 but between 2 kilometers of the CCZ and 4 kilometers from the boundary of the CCZ and identify this as spillover region 2. The monthly accidents within each of these spillover zones is then used in place of those in the CCZ in a model that otherwise replicates Table 1 by comparing them to the accidents in the 20 largest English cities during the congestion charge times. As the first two columns of Table 2 show, there is no evidence of substitution. Not only does the number of accidents in these two regions fail to increase as a result of the congestion charge, but they significantly decrease. These effects are sizeable, 18-20 less accidents per month in each of these spillover areas. Thus, the response to congestion charge would appear to be a reduced number of journeys through these areas into central London or an increase in the number of people who travel through these areas by mass transit. In either case, the reduction in accidents within the CCZ is clearly an underestimate of the full number of accident reductions.

We next examine what happened to the number of accidents occurring outside of the business hours, five days a week, in which the charge is levied. Again, trips that might have happened at these peak times (for shopping for example) may simply be postponed till later in the evening or the weekend. This would also cause a displacement in accidents rather than a reduction. Column 3 compares the accidents in the CCZ but out of charged hours to the accidents in the 20 largest cities out of charged hours. There is no evidence of displacement and, indeed, the out of hours accidents in the CCZ actually decline significantly relative to the control. The 
congestion charge is associated with fewer accidents not only in the charged zone and time but outside the charged zone (but nearby) and outside of the charged time.

Finally, we examine the accidents in the CCZ and charged hours that involve at least one "uncharged" vehicle. Again, commuters can substitute away from charged automobiles to these taxis, motorcycles and bicycles. These accidents are compared to accidents that involve at least one uncharged vehicle during the charged hours in the 20 largest cities. Again our estimates find a marked reduction in accidents involving these vehicles. This may reflect fewer automobiles on the road and that this decreases the odds of the uncharged vehicles being in an accident even if the number of uncharged vehicles actually increased.

We emphasize that while the estimates in Table 2 simply retain the 20 largest cities as the control in the tests of the spillover effect, the results are robust to the matching procedure. In estimates available from the authors, we created a new synthetic control for each spillover examination (two on area and one each on time and vehicle type). The estimated coefficients on the difference-in-difference are very similarly sized to those in Table 2 and indicate in each case a significant reduction in the number of accidents in targeted spillover relative to the relevant synthetic control.

The critical point is that we have found no evidence of substitution in which uncharged adjacent areas, hours or vehicles have increased accidents as a result of the congestion charge. Instead, the influence of the congestion charge appears substantially larger than would be indicated by limiting the analysis to the zone, time and vehicles directly charged. Indeed, the reduction in accidents in the charged zone, time and vehicles is actually smaller than the sum of reductions in other areas, times and vehicles. Thus, there seems to have been a more general and fundamental change in the number of trips and/or mode of transportation. 


\section{INSERT TABLE 3}

\section{Serious Injuries and Fatalities}

While the reduction in accidents is large and widespread, it is not necessarily the case that this translates into a lower incidence of accidents involving severe injury or death. As discussed, the higher road speeds associated with the congestion charge may increase the severity of the accidents that do occur. Minor accidents at a slow speed can involve serious injuries or death at a higher speed. Moreover, the potential substitution towards vehicles with a greater inherent danger of serious injury, such as more accidents involving automobiles and bicycles, also suggests that even though there may be fewer accidents there may be more accidents with serious consequences. In addition to this ambiguity, examining accidents that involve hospitalization and death are critical for at least two reasons. First, such accidents likely constitute the bulk of the social costs associated with traffic accidents and so are of strong policy interest. Second, such accidents are subject to less measurement error as they are much more likely to be reported and recorded in the administrative statistics.

Table 3 examines the influence of the congestion charge by re-estimating our main model for all serious and fatal accidents and then for only fatal accidents. The estimates are limited to the CCZ and for accidents involving a charged vehicle in charged times. They are, of course, smaller in absolute terms as serious and fatal accidents happen less frequently than do all

accidents. Yet, they still remain negative and statistically significant. Moreover, in percentage terms these emerge as very large effects. The estimates indicate that the congestion charge reduced the number of serious and fatal accidents in the congestion zone by 46 a year and reduced the number of fatalities by 4.6 a year. 
Again, the issue of spillovers and substitution can be critical in assessing the full influence of the congestion charge. We reproduce the estimates in the two adjacent uncharged regions, for the uncharged hours and for uncharged vehicles within charged hours. In each case the number of serious and fatal accidents falls relative to similarly constructed controls. The declines remain large and significant with percentage declines ranging from 8 to 25 percent. As in the case of all accidents, failure to recognize the reductions in adjacent areas, times and uncharged vehicles would grossly underestimate the true influence of the congestion charge on serious and fatal accidents.

\section{INSERT TABLE 5}

A particular concern of policy makers has been the hazard faced by bicycle riders. Indeed, Li et al. (2012) suggest increased bike ridership as a result of the congestion charge led to an increase in accidents and serious injuries by those on bikes. We return to this using our preferred specification and limiting our dependent variable to accidents involving bikes. These results are reported in Table 5 where we provide estimates for all accidents and for serious and fatal accidents. Critically, we use our longer evaluation window rather than the short window ending with 2005 as done in the previous study. Contrary to that previous evidence, we find a reduction in bike accidents that fits with the evidence for other types of spillovers.

In an attempt to reconcile our results with this previous evidence we limit our estimates to successively smaller post-policy periods. Reducing our period to the end of 2006 substantially reduces the size of the policy effect, and further trimming the period to the end of 2005 recovers the congestion charge increasing bike accidents, both overall and for serious and fatal accidents. Thus, an appropriate summary would be there existed a short-term increase in bike accidents that 
dissipated and reversed. This fits with new inexperienced bicycle commuters initially flooding the congestion zone. Yet, this eventually became dominated by the underlying lower probability of traffic accidents as the riders either gained experience and ability as commuters or found alternative modes of transport.

\section{The Effect of Later Policy Changes}

Our last step is to examine two additional sources of variation in the original congestion charge policy intervention. In the first source of variation we recognize that the original congestion charge was set at $£ 5$ but that this was subsequently increased to $£ 8$ in $2008 .{ }^{6}$ We use this information, in combination with variation in the consumer price index, to generate an annual real congestion zone charge in 2003 pounds. In the first column of Table 6 we replace the dummy variable for the CCZ with this real congestion zone charge as a measure of policy intensity. The resulting estimate indicates that each real pound in the charge causes a reduction of just under 6 accidents per month in the charged area.

\section{INSERT TABLE 6}

The second major source of variation was a temporary extension of the original congestion charge zone to incorporate more western areas (the so-called western extension). This extension occurred on February 17, 2007 but charging for the extension was removed on December 24, 2010. Mayor Boris Johnson was quoted shortly after the removal saying that the removal did not substantially increase congestion and "there has been no significant downside in removing the western extension zone (London24)." In part this may reflect that the extension

\footnotetext{
${ }^{6}$ There was an additional charge increase 2011 that is outside the time frame of our evaluation window.
} 
always included "free through routes" that were never charged. Also, in part, our previous analysis of spatial spillovers suggests that adjacent areas, including the western extension, were, in effect, already partially treated. The traffic through this area was reduced by the initial congestion zone charge.

\section{INSERT TABLE 7}

We test the consequences of the Western Extension in two related fashions. For each test we extend our original model to incorporate the Western Extension as a treatment area (mapped by GIS and matched to traffic accidents). In the first test we include both the CCZ and the Western Extension in a treatment indicator that turns on for the CCZ in February 2003 and on for the Western Extension in February 2007. Note that our data window ends at the same time as the Western Extension is removed. In the second test we imagine two separate treated areas, the CCZ and the Western Extension and have two separate difference-in difference estimates. To allow for the complicated dynamics associated with the likely spillovers, we include trends for the control (the other English cities), the CCZ and the Western Extension for both the pretreatment and treatment period.

As shown in the first column of Table 7, the first test suggests that the treatment on the two combined regions is associated with a highly significant reduction of 41.5 accidents per period. The second test in column 2 suggests that the implementation of the CCZ is associated with a highly significant decline of 46 accidents within the CCZ and that the implementation of the Western Extension is associated with a significant but more modest decline of 13.5 accidents per period within the Extension. The critical point from our perspective is that there is no evidence to suggest that the implementation of the Western Extension is somehow inappropriately generating the fundamental results we have been showing for the CCZ. 


\section{Conclusion}

In what has been hailed as a triumph of economics, London has for the last decade charged drivers to enter the central congestion zone. While some other cities, including Singapore and Manchester, have either implemented or considered such congestion charges there has not yet been a huge movement to mimic London. The advantages of reduced congestion include improved travel times and reliability, reduced air pollution from vehicles stuck in traffic and, potentially fewer traffic accidents and lost lives. Theoretically the increased speed may work to mitigate reduced congestion by increasing accidents and their severity and substitution away from the charged zone, hours and vehicles may also reduce or eliminate any net reduction in accidents.

We have undertaken a comprehensive examination of the consequences of the London congestion charge on vehicle accidents. We find a substantial and significant reduction in the number of accidents in the charged zone for charged vehicles and times relative to sensible controls. This persists for serious and for fatal accidents. Critically, there is no evidence that the congestion charge resulted in a permanent increase in accidents for uncharged times, adjacent geographic regions or uncharged vehicles. Indeed, we find evidence reductions and these results also persist for serious and for fatal accidents.

We view this evidence as broadly consistent with the intention of the government to use the congestion charge as a mechanism to move travelers from automobiles to public transit. On the one hand, the charge discouraged the use of automobiles and, on the other hand, the funds raised by the charge were spent on improving public transit, largely bus lines. The public transit 
authorities did, indeed, see a large increase in bus ridership (Transit for London 2004). While we have focused on only one benefit from the charge, accident reduction, other benefits include increased speed, travel reliability and reduced air pollution. Obviously, important distributional aspects have also not been examined. There be movements away from central city shops and entertainment as an example. Nonetheless, we provide the most comprehensive examination of the influence of the charge on traffic accidents and find important reductions in lost money and lives. 


\section{References}

Abadie, Alberto, Alexis Diamond and Jens Hainmueller. 2010."Synthetic Control Methods for Comparative Case Studies: Estimating the Effect of California’s Tobacco Control Program," Journal of the American Statistical Association 105: 493-505.

Alberto Abadie and Javier Gardeazabal. 2003. "The Economic Costs of Conflict: A Case Study of the Basque Country," American Economic Review 93: 113-132.

Ashenfelter, Orley and Michal Greenstone. 2004. "Using Mandates Speed Limits to Measure the Value of a Statistical Life," Journal of Political Economy 112: s226 - s267.

Cameron, A.C., Gelbach, J.B., Miller, D.L., 2008. “Bootstrapped-based Improvements for Inference with Clustered Errors,” Review of Economics and Statistics 90: 414-427.

Conley, Timothy G., and Christopher R. Taber, 2011. "Inference with “difference in differences” with a small number of policy changes." The Review of Economics and Statistics 93: 113125.

Fosgerau, Morgens and André de Palma. 2013. "The Dynamics of Urban Traffic Congestion and the Price of Parking," Journal of Public Economics 105: 106-115.

Department of Transport (2012) Reported Road Casualties Great Britain: Annual Report 2011, London.

Hårsman, Bjorn and John M. Quigley. 2010. "Political and Public Acceptability of Congestion Pricing: Ideology and self-interest," Journal of Policy Analysis and Management 29: 854874.

Leape, Jonathan. 2006. "The London Congestion Charge," Journal of Economic Perspectives 20: $157-76$. 
Li, Jaojie, Daniel J. Graham, Arnab Majumdar. 2012. "The Effects of Congestion Charring on Road Traffic Casualties: A Causal Analysis Using Difference-in-Difference Estimation," Accident Analysis and Prevention 49: 366 - 77.

London24, "West London Congestion Zone Axe Brings Positive Results” http://www.london24.com/news/transport/west_london_congestion_charge_zone_axe_bring s_positive_results_1_912962 (viewed on June 29, 2014).

Newberry, David M. 1990. "Pricing and Congestion: Economic Principles Relevant to Pricing Roads," Oxford Review of Economic Policy 6: 22- 38.

Parry, Ian WH, and Antonio Bento. 2002. "Estimating the welfare effect of congestion taxes: the critical importance of other distortions within the transport system." Journal of Urban Economics 51: 339-365.

Parry, Ian W. H., Margaret Walls and Winston Harrington. 2007. "Automobile Externalities and Policies," Journal of Economic Literature 45: 373-399.

Pigou, A. C. 1924. The Economics of Welfare. Transaction Publishers, New York.

Quddus, M.A. 2008. "Time Series Count Data Models: An Empirical Application to Traffic Accidents," Accident Analysis and Prevention 40: 1732-1741.

Shefer, D. and Rietveld, P. 1997. "Congestion and Safety on Highways: Towards an Analytical Model," Urban Studies 34: 679-92.

Shepherd, Simon, and Agachai Sumalee. 2004."A Genetic Algorithm Based Approach to Optimal Toll Level and Location Problems," Network and Spatial Economics 4: 161-79.

Small, Kenneth A., Clifford Winston, and Jia Yan. 2005. "Uncovering the Distribution of Motorists’ Preferences for Travel Time and Reliability," Econometrica 73:4, pp, 1367-82. Transport for London. 2003. Congestion Charging: Six Months On. London, June. 
Transport for London. 2005. Central London Congestion Charging Scheme Impacts Monitoring: Summary Review. London, January.

Verhoef, Erik and Jan Rouwendel. 2004. "A Behavioural Model of Traffic Congestion:

Endogenizing Speed choice, Traffic safety and Time losses," Journal of Urban Economics 56: $408-34$.

Vickrey, William. 1963. "Pricing in Urban and Suburban Transport," American Economic Review 52: 452-65.

Walters, Alan A. 1961. "The Theory and Measurement of Private and Social Cost of Highway Congestion.” Econometrica. 29: 676-99.

Wang, Chao, Quddus, Mohammed A. and Ison, Stephen G. 2009. "Impact of Traffic Congestion on Road Accidents: A Spatial analysis of the M25 Motorway in England," Accident Analysis and Prevention 41: 798 - 808. 
Figure 1: The original London Congestion Charge Zone

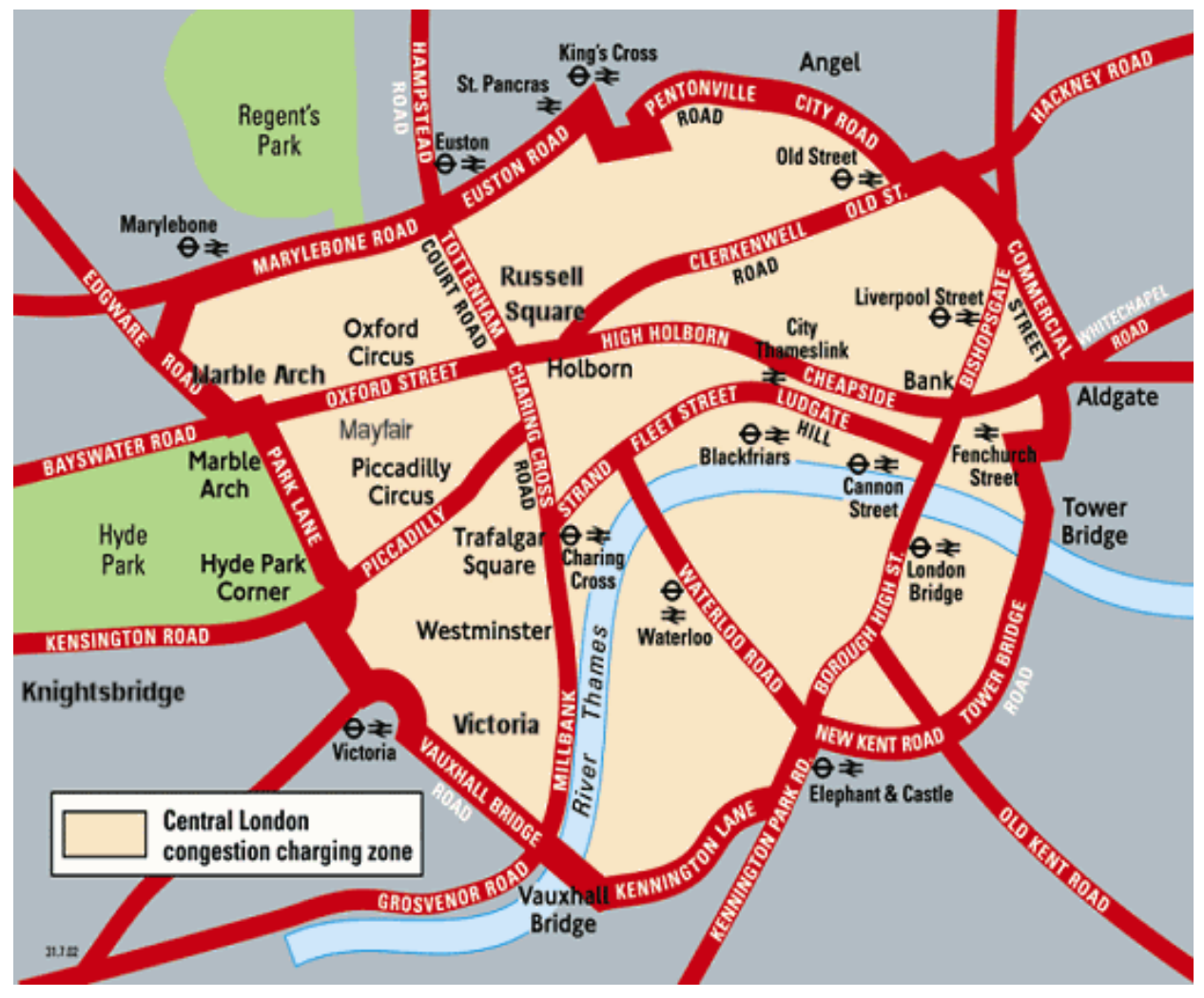


Figure 2: Accidents involving charged vehicles in charged times, CCZ vs the 20 largest cities in Great Britain

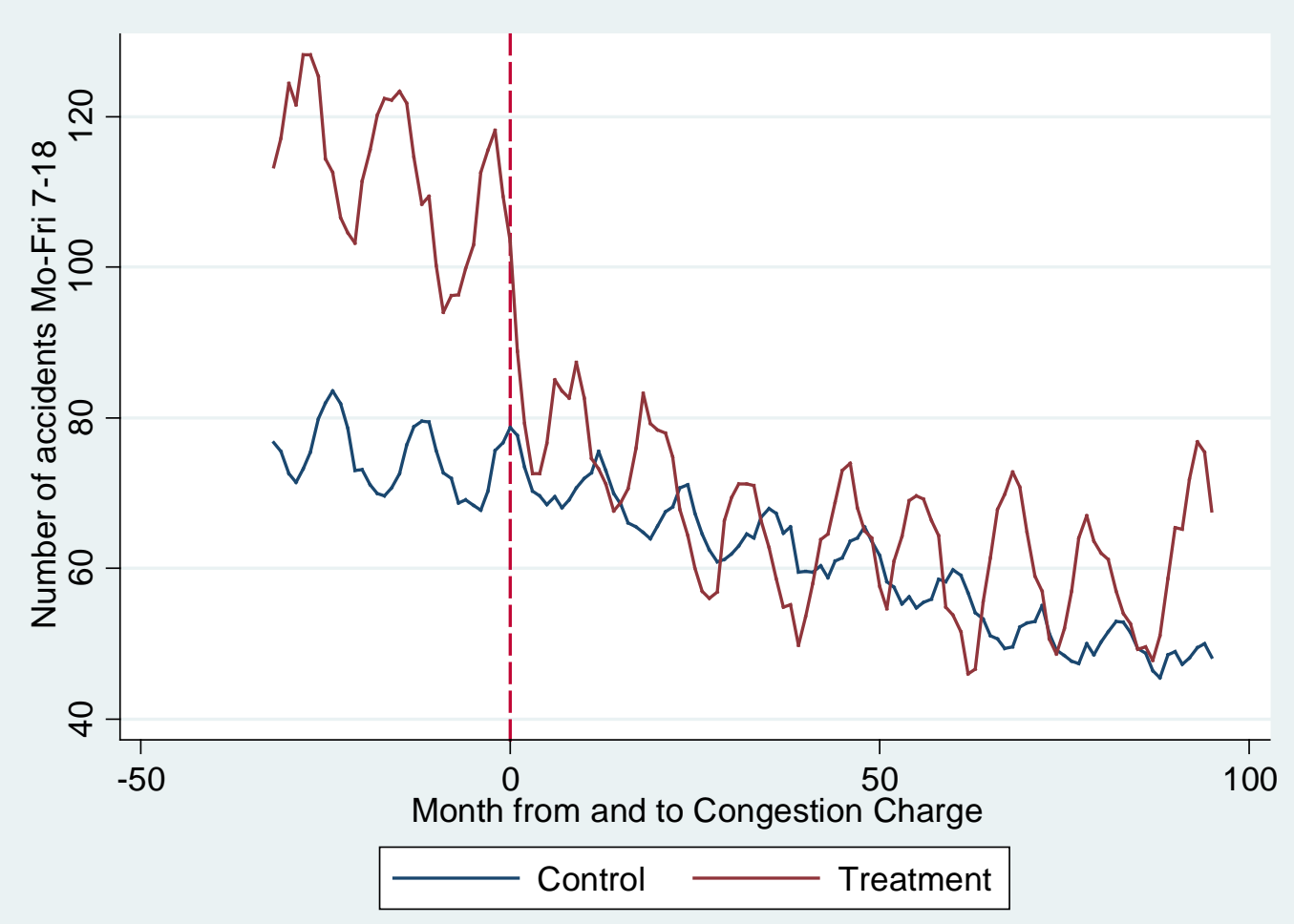


Figure 3: Spline Regression for Charged vehicles, charged hours in CCZ vs the 20 largest cities in Great Britain

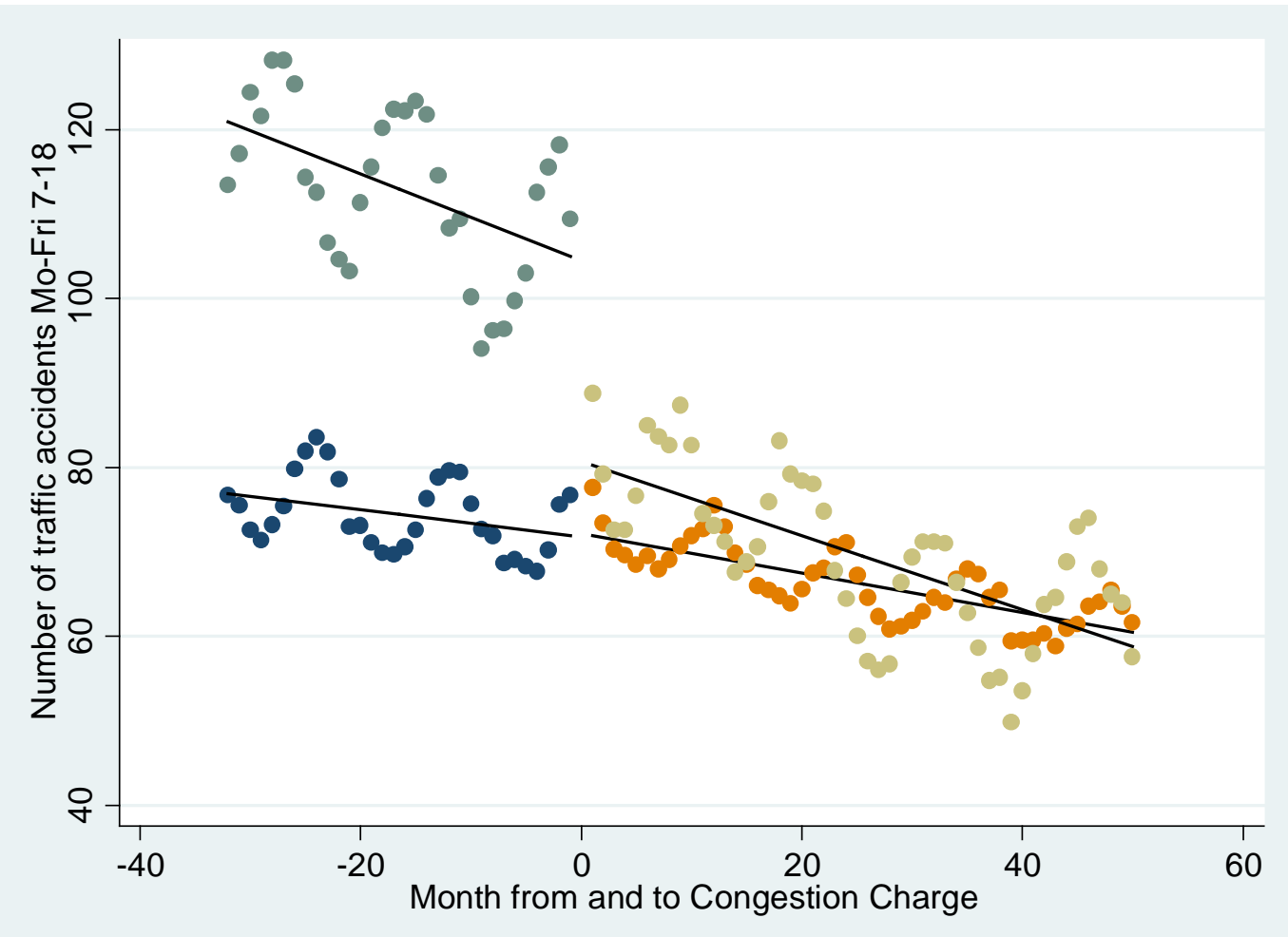


Figure 4: Synthetic Cohort Estimates of Congestion Charge Effect on Traffic Accidents

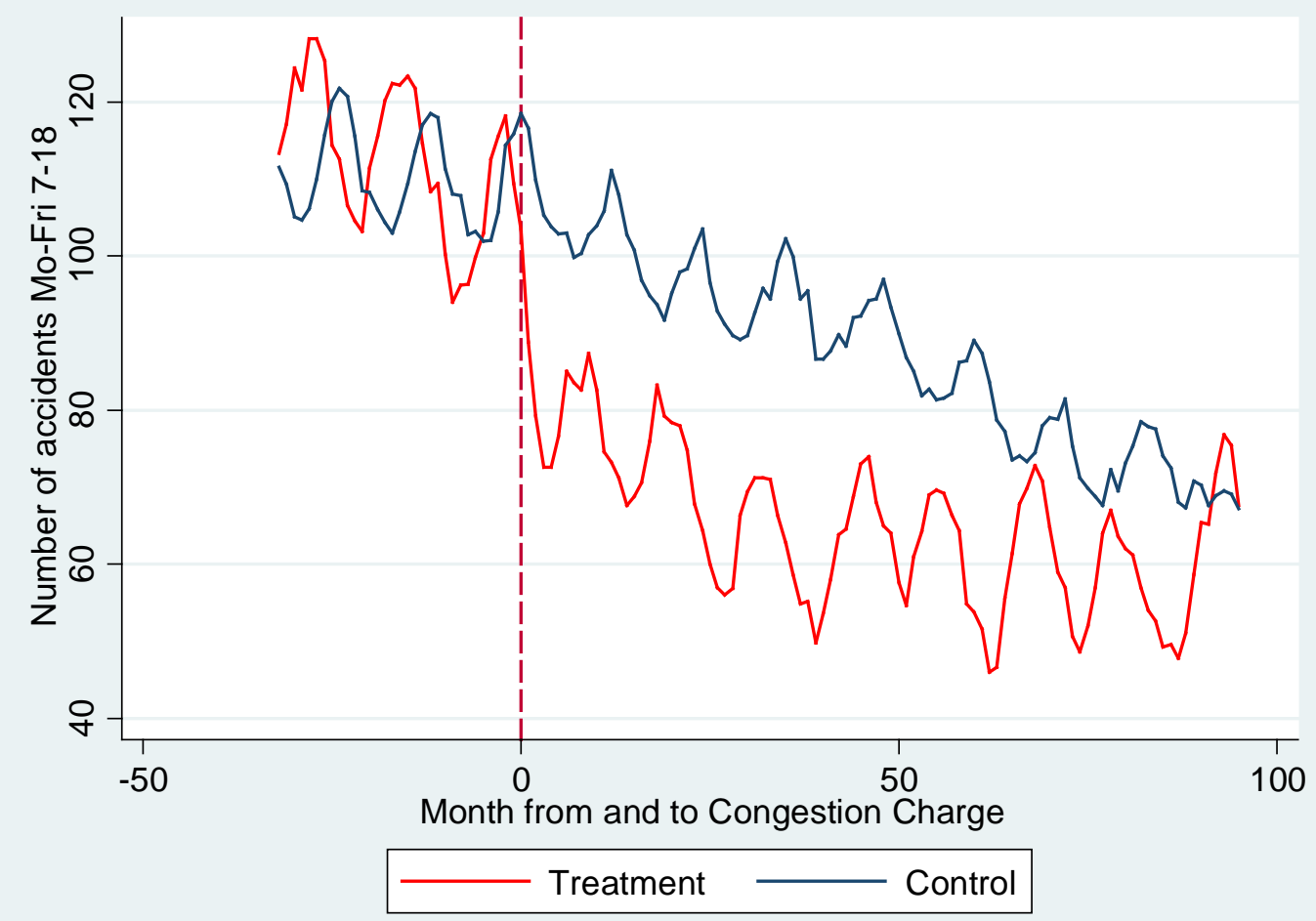


TABLE 1: Effect of Congestion Charges on Monthly Accidents for Charged Vehicles in Charged Times in the CCZ vs Charged Vehicles and Times in the 20 Largest British Cities, 2000-2010

\begin{tabular}{cccccc}
\hline & $\mathbf{( 1 )}$ & $\mathbf{( 2 )}$ & $\mathbf{( 3 )}$ & $\mathbf{( 4 )}$ & $\mathbf{( 5 )}$ \\
\hline \multirow{2}{*}{ CCZ*Policy } & -30.254 & -34.148 & -0.385 & -0.403 & -33.911 \\
& $(2.554)^{* * *}$ & $(0.910)^{* * *}$ & $(0.014)^{* * *}$ & $(0.014)^{* * *}$ & $(5.576)^{* * *}$ \\
Policy & 2.025 & 2.210 & 0.059 & 0.062 & 1.914 \\
& $(0.935)^{* *}$ & $(0.931)^{* *}$ & $(0.014)^{* * *}$ & $(0.014)^{* * *}$ & $(3.145)$ \\
CCZ & 36.096 & 34.975 & 0.368 & 0.364 & -3.426 \\
& $(9.078)^{* * *}$ & $(9.752)^{* * *}$ & $(0.123)^{* * *}$ & $(0.123)^{* * *}$ & $(3.454)$ \\
Month Trend & -0.276 & -0.279 & -0.005 & -0.005 & -0.408 \\
& $(0.038)^{* * *}$ & $(0.040)^{* * *}$ & $(0.000)^{* * *}$ & $(0.000)^{* * *}$ & $(0.033)^{* * *}$ \\
Month Trend * CCZ & & 0.059 & 0.001 & 0.002 & 0.186 \\
& & $(0.038)$ & $(0.000)^{* * *}$ & $(0.000)^{* * *}$ & $(0.062)^{* * *}$ \\
Quarter 1 & -9.252 & -9.252 & -0.143 & -0.142 & -11.560 \\
& $(1.244)^{* * *}$ & $(1.245)^{* * *}$ & $(0.009)^{* * *}$ & $(0.009)^{* * *}$ & $(2.158)^{* * *}$ \\
Quarter 2 & -8.488 & -8.488 & -0.131 & -0.128 & -3.869 \\
& $(1.278)^{* * *}$ & $(1.278)^{* * *}$ & $(0.014)^{* * *}$ & $(0.013)^{* * *}$ & $(2.200)^{*}$ \\
Quarter 3 & -8.135 & -8.135 & -0.125 & -0.122 & -2.158 \\
& $(1.435)^{* * *}$ & $(1.435)^{* * *}$ & $(0.017)^{* * *}$ & $(0.015)^{* * *}$ & $(2.371)$ \\
Constant & 86.726 & 86.779 & 4.505 & 4.504 & 123.256 \\
& $(10.540)^{* * *}$ & $(10.572)^{* * *}$ & $(0.124)^{* * *}$ & $(0.124)^{* * *}$ & $(2.666)^{* * *}$ \\
Observations & 2772 & 2772 & 2772 & 2772 & 264 \\
R-squared & 0.10 & 0.10 & & & 0.74 \\
\hline
\end{tabular}

Robust standard errors clustered at a local authority level in parentheses. ***,**, * indicate statistical significance at the $1 \%, 5 \%$ and $10 \%$ level, respectively. 
TABLE 2: Spillover Effects of the Congestion Charge on Accidents vs. 20 Largest British Cities

\begin{tabular}{ccccc}
\hline & $\mathbf{( 1 )}$ & $\mathbf{( 2 )}$ & $\mathbf{( 3 )}$ & $\mathbf{( 4 )}$ \\
\hline & Spillover Region 1 & Spillover Region 2 & Not Charged Time & Not Charged Vehicles \\
CCZ*Policy & -18.316 & -20.498 & -15.777 & -20.693 \\
& $(0.910)^{* * *}$ & $(0.910)^{* * *}$ & $(0.834)^{* * *}$ & $(0.675)^{* * *}$ \\
& {$[-0.193]$} & {$[-0.176]$} & {$[-0.219]$} & {$[-0.201]$} \\
Policy & 2.221 & 2.229 & 0.530 & 0.792 \\
& $(0.931)^{* *}$ & $(0.931)^{* *}$ & $(0.862)$ & $(0.683)$ \\
CCZ & 44.061 & 82.722 & 28.420 & 111.200 \\
& $(9.752)^{* * *}$ & $(9.752)^{* * *}$ & $(7.978)^{* * *}$ & $(2.425)^{* * *}$ \\
Month Trend & -0.279 & -0.279 & -0.183 & -0.070 \\
& $(0.040)^{* * *}$ & $(0.040)^{* * *}$ & $(0.030)^{* * *}$ & $(0.011)^{* * *}$ \\
Month Trend * CCZ & -0.049 & -0.121 & -0.009 & -0.366 \\
& $(0.038)$ & $(0.038)^{* * *}$ & $(0.028)$ & $(0.011)^{* * *}$ \\
Quarter Dummies & $\mathrm{V}$ & $\mathrm{V}$ & $\mathrm{V}$ & $\mathrm{V}$ \\
& & & & \\
Constant & 86.881 & 86.905 & 66.649 & 25.952 \\
& $(10.569)^{* * *}$ & $(10.569)^{* * *}$ & $(8.632)^{* * *}$ & $(2.580)^{* * *}$ \\
Observations & 2772 & 2772 & 2772 & 2772 \\
R-squared & 0.12 & 0.20 & 0.08 & 0.69 \\
\hline
\end{tabular}

Robust standard errors clustered at a local authority level in parentheses. [] provide estimates from Negative Binomial regressions for purposes of comparison. ***,**, * indicate statistical significance at the 1\%, 5\% and 10\% level, respectively. 
TABLE 3: Serious and Fatal Injuries and Congestion Charging

\begin{tabular}{cll}
\hline & $\begin{array}{l}\text { Serious and } \\
\text { Fatal }\end{array}$ & Fatalities \\
\hline & & \\
CCZ*Policy & -3.841 & -0.387 \\
& $(0.236)^{* * *}$ & $(0.031)^{* * *}$ \\
& {$[-0.300]$} & {$[-0.821]$} \\
Policy & -0.222 & -0.000 \\
& $(0.229)$ & $(0.029)$ \\
CCZ & 7.245 & 0.246 \\
& $(1.011)^{* * *}$ & $(0.059)^{* * *}$ \\
Month Trend & -0.020 & -0.002 \\
& $(0.003)^{* * *}$ & $(0.000)^{* * *}$ \\
Month Trend * CCZ & -0.010 & 0.002 \\
& $(0.003)^{* * *}$ & $(0.000)^{* * *}$ \\
Quarter Dummies & $\mathrm{V}$ & $\mathrm{V}$ \\
Constant & 8.561 & 0.510 \\
& $(1.109)^{* * *}$ & $(0.073)^{* * *}$ \\
Observations & 2772 & 2772 \\
R-squared & 0.09 & 0.02 \\
\hline
\end{tabular}

Robust standard errors clustered at a local authority level in parentheses. [] provide estimates from Negative Binomial regressions for purposes of comparison. ***,**, * indicate statistical significance at the 1\%, 5\% and 10\% level, respectively. 
Table 4: Spillovers in Serious and Fatal Accidents

\begin{tabular}{cllll}
\hline & \multicolumn{2}{c}{$(\mathbf{1})$} & \multicolumn{1}{c}{$(\mathbf{2})$} & \multicolumn{1}{c}{$(\mathbf{3})$} \\
\hline & Spillover Region 1 & Spillover Region 2 & Not Charged Time & Not Charged Vehicles \\
CCZ*Policy & -3.653 & & & \\
& $(0.236)^{* * *}$ & -2.241 & -2.480 & -2.048 \\
& {$[-0.250]$} & $(0.236)^{* * *}$ & $(0.296)^{* * *}$ & $(0.139)^{* * *}$ \\
Policy & -0.321 & {$[-0.079]$} & {$[-0.239]$} & {$[-0.115]$} \\
& $(0.236)$ & -0.321 & -0.198 & 0.010 \\
CCZ & 8.751 & $(0.236)$ & $(0.296)$ & $(0.139)$ \\
& $(1.010)^{* * *}$ & 14.451 & 4.428 & 15.254 \\
Month Trend & -0.018 & $(1.010)^{* * *}$ & $(1.164)^{* * *}$ & $(0.358)^{* * *}$ \\
& $(0.003)^{* * *}$ & -0.018 & -0.025 & -0.007 \\
Month Trend * CCZ & -0.010 & $(0.003)^{* * *}$ & $(0.005)^{* * *}$ & $(0.002)^{* * *}$ \\
& $(0.003)^{* * *}$ & -0.047 & 0.005 & -0.056 \\
Quarter Dummies & $(0.003)^{* * *}$ & $(0.005)$ & $(0.002)^{* * *}$ \\
& 7.761 & 7.761 & 8.703 & 3.173 \\
Constant & $(1.010)^{* * *}$ & $(1.010)^{* * *}$ & $(1.164)^{* * *}$ & $(0.358)^{* * *}$ \\
& 2772 & 2772 & 2772 & 2772 \\
& 0.11 & 0.22 & 0.06 & 0.48 \\
\hline
\end{tabular}

Robust standard errors clustered at a local authority level in parentheses. [] provide estimates from Negative Binomial regressions for purposes of comparison. ***,**, * indicate statistical significance at the 1\%, 5\% and 10\% level, respectively. 
Table 5: Congestion Charge and Bike Accidents

\begin{tabular}{cllllll}
\hline & \multicolumn{3}{c}{ All Accidents } & \multicolumn{2}{c}{ Serious and Fatal Accidents } \\
\hline & \multicolumn{1}{c}{ Bikes } & $\mathbf{2 0 0 0 - 2 0 0 6}$ & $\mathbf{2 0 0 0 - 2 0 0 5}$ & \multicolumn{1}{c}{ Bikes } & $\mathbf{2 0 0 0 - 2 0 0 6}$ & $\mathbf{2 0 0 0 - 2 0 0 5}$ \\
& & & & & & \\
CCZ*Policy & -5.164 & -1.505 & 1.429 & -0.481 & 0.156 & 0.990 \\
& $(0.247)^{* * *}$ & $(0.302)^{* * *}$ & $(0.303)^{* * *}$ & $(0.063)^{* * *}$ & $(0.106)$ & $(0.099)^{* * *}$ \\
Policy & -0.782 & -0.293 & 0.041 & -0.077 & -0.070 & -0.032 \\
& $(0.240)^{* * *}$ & $(0.286)$ & $(0.297)$ & $(0.062)$ & $(0.106)$ & $(0.097)$ \\
CCZ & 23.833 & 25.497 & 27.510 & 3.002 & 3.371 & 3.970 \\
& $(0.710)^{* * *}$ & $(0.718)^{* * *}$ & $(0.740)^{* * *}$ & $(0.082)^{* * *}$ & $(0.081)^{* * *}$ & $(0.084)^{* * *}$ \\
Month Trend & 0.006 & -0.007 & -0.019 & 0.003 & 0.003 & 0.002 \\
& $(0.003)$ & $(0.007)$ & $(0.008)^{* *}$ & $(0.001)^{* * *}$ & $(0.002)$ & $(0.002)$ \\
Month Trend * CCZ & 0.065 & -0.022 & -0.128 & 0.010 & -0.009 & -0.041 \\
& $(0.003)^{* * *}$ & $(0.007)^{* * *}$ & $(0.007)^{* * *}$ & $(0.001)^{* * *}$ & $(0.002)^{* * *}$ & $(0.002)^{* * *}$ \\
Quarter Dummies & & & & & & \\
Constant & 6.644 & 6.947 & 7.228 & 0.643 & 0.676 & 0.687 \\
& $(0.780)^{* * *}$ & $(0.813)^{* * *}$ & $(0.862)^{* * *}$ & $(0.100)^{* * *}$ & $(0.110)^{* * *}$ & $(0.113)^{* * *}$ \\
Observations & 2772 & 1764 & 1512 & 2772 & 1764 & 1512 \\
R-squared & 0.56 & 0.57 & 0.57 & 0.31 & 0.29 & 0.29 \\
\hline
\end{tabular}

Robust standard errors clustered at a local authority level in parentheses. ***,**, * indicate statistical significance at the 1\%, 5\% and 10\% level, respectively. 
Table 6: Prices, Congestions Charges and Traffic Accidents

\begin{tabular}{cc}
\hline & Price Variation \\
\hline CCZ*Price (£) & -5.757 \\
& $(0.162)^{* * *}$ \\
Price (£) & 0.382 \\
& $(0.166)^{* *}$ \\
CCZ & 31.855 \\
& $(9.743)^{* * *}$ \\
Month Trend & -0.286 \\
& $(0.042)^{* * *}$ \\
Month Trend $*$ CCZ & 0.156 \\
& $(0.040)^{* * *}$ \\
Constant & 86.981 \\
& $(10.580)^{* * *}$ \\
Observations & 2772 \\
R-squared & 0.10 \\
\hline
\end{tabular}

Robust standard errors clustered at a local authority level in parentheses. ***,**, * indicate statistical significance at the 1\%, 5\% and 10\% level, respectively. All models include quarterly dummies. 
Table 7: The Impact of the Western Extension on Traffic Accidents.

\begin{tabular}{ccc}
\hline & (I) & (II) \\
\hline Treatment*Policy & $-42.401^{* * *}$ & \\
CCZ*Policy 2003 & $(5.922)$ & \\
& & $-46.763^{* * *}$ \\
Western Extension*Policy 2007 & & $(2.123)$ \\
Policy 2003 & & $-13.543^{* * *}$ \\
& 1.268 & $(2.913)$ \\
Policy 2007 & $(2.754)$ & 1.466 \\
& 1.033 & $(2.704)$ \\
CCZ & $(3.917)$ & -0.279 \\
& $42.752^{* * *}$ & $(3.728)$ \\
Western Extension & $(9.513)$ & $44.971^{* * *}$ \\
& $-29.779 * * *$ & $-30.700^{* * *}$ \\
Month Trend & $(9.470)$ & $(9.489)$ \\
& $-0.176^{* * *}$ & $-0.173^{* * *}$ \\
Month Trend $*$ CCZ & $(0.059)$ & $(0.058)$ \\
& $-0.378^{* * *}$ & $-0.468^{* * *}$ \\
Month Trend * Western Extension & $(0.133)$ & $(0.060)$ \\
& 0.008 & 0.024 \\
Observations & $(0.034)$ & $(0.034)$ \\
R-squared & & \\
\end{tabular}

Robust standard errors clustered at a local authority level in parentheses. ***,**, * indicate statistical significance at the 1\%,5\% and 10\% level, respectively. All models include quarterly dummies.

Treatment*Policy is a dummy variable which takes value 1 for the initial CCZ from the $17^{\text {th }}$ of February 2003 and for the Western Extension from the $19^{\text {th }}$ of February 2007 onwards and 0 otherwise. CCZ corresponds to the initial congestion charge zone and Western Extension corresponds to the extended area. Policy 2003 takes value 1 from the $17^{\text {th }}$ of February 2003 and 0 otherwise. Policy 2007 takes value 1 from February 2007 and 0 otherwise. 


\section{APPENDIX}

Appendix Table A: Robustness Checks

\begin{tabular}{cccc}
\hline & $(\mathrm{I})$ & $(\mathrm{II})$ & (III) \\
\hline & Density & Population \& & Trimmed Sample \\
& Control & Area controls & $(2000-2006)$ \\
CCZ*Policy & -43.724 & -33.402 & -20.345 \\
& $(24.504)^{*}$ & $(1.532)^{* * *}$ & $(1.346)^{* * *}$ \\
Policy & 0.883 & -3.210 & 0.257 \\
& $(3.408)$ & $(1.216)^{* *}$ & $(1.216)$ \\
CCZ & 28.776 & 60.527 & 41.756 \\
& $(22.104)$ & $(3.240)^{* * *}$ & $(9.337)^{* * *}$ \\
Month Trend & -0.252 & -0.067 & -0.225 \\
& $(0.064)^{* * *}$ & $(0.017)^{* * *}$ & $(0.034)^{* * *}$ \\
Month Trend ${ }^{*}$ CCZ & 0.106 & -0.081 & -0.298 \\
& $(0.139)$ & $(0.021)^{* * *}$ & $(0.030)^{* * *}$ \\
Population & & 0.365 & \\
& & $(0.045)^{* * *}$ & \\
Area & & 0.003 & \\
& & $(0.001)^{* *}$ & \\
Density & 0.165 & & \\
& $(0.421)$ & $\mathrm{V}$ & \\
Quarter Dummies & $\mathrm{V}$ & $\mathrm{V}$ & $\mathrm{V}$ \\
Constant & 81.960 & 46.364 & $(10.359)^{* * *}$ \\
& $(11.767)^{* * *}$ & $(3.221)^{* * *}$ & 1764 \\
Observations & 2772 & 2772 & 0.05 \\
R-squared & 0.11 & 0.86 & \\
\hline
\end{tabular}

